\title{
A CASE OF MOYA MOYA DISEASE PRESENTING AS SEIZURE AND HEMIPARERSIS
}

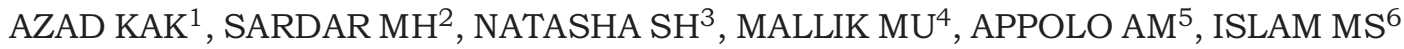

\begin{abstract}
:
This is a case of a twelve year old boy presenting with right sided weakness, inability to speak and one episode of seizure. On admission he had pure motor aphasia, right sided hemiparersis with a muscle power of $1 / 5$ and his GCS was 8. Initially it was thought to be a case of intra cranial space occupying lesion with a differential diagnosis of vasculitis. Finally digital subtraction angiogram revealed it to be a case of moyamoya Disease. Moyamoya Disease is a rare disease with a genetic abnormality and unilateral presentation is even rarer.
\end{abstract}

Key words: Moyamoya disease, seizure, hemiparersis.

J Dhaka Med Coll. 2011; 20(2) : 218-221.

\section{Introduction:}

Moyamoya Disease, first described by Takeuchi and Shimizu ${ }^{1}$, is a rare cerebrovascular condition characterized by progressive stenosis of bilateral internal carotid arteries with compensatory formation of extensive collateral circulation by the dural, leptomeningeal and other perforating blood vessels giving rise to the typical "puff of smoke" appearance in angiogram. The term moyamoya is a Japanese word which means "a puff of smoke". It was coined by Suzuki and Takaku in 1969 to describe the angiographic appearance of the collateral vessels at the base of the brain in a group of 21 patients with internal carotid arterial occlusion. ${ }^{2}$ The disease process is characterized by progressive bilateral stenosis on intracranial internal carotid arteries that eventually affects its major two branches, the anterior and middle cerebral artery. Posterior circulation involvement has also been noted in advanced cases with particular preponderance of forming aneurysms.

The histopathology of the affected arteries demonstrate a fibrocellular thickening of the intima, proliferated smooth muscle cells, prominently tortuous and often duplicates internal elastic lamina, with no inflammatory or atheromatous involvement. Moyamoya disease is 1.8 times more common in women and the highest incident is found in population of Asia, specially Japan where the incidence rate is $0.35-2$ per $100,000 .{ }^{4}$ Moyamoya disease has a bimodal presentation with ischaemic events being more common in young age and haemorrhagic events more common after the fourth decade of life. Arteriography and Angiogram are the gold standard of diagnosis and as a result this disease may remain underdiagnosed in many centres where these facilities are unavailable.

\section{Case Report:}

A 12 year old right handed boy was admitted to the Department of Medicine, Dhaka Medical College Hospital (DMCH), Dhaka, with the complaints of right sided weakness of the body and inability to speak for 4 days, seizure associated with vomiting for one episode. According to his attendants, he was reasonably well about 4 days back then he suddenly developed inability to speak with right sided weakness of whole body. He was at a boarding school at the time of the incidence. Then he developed convulsion one day after this event

1. Prof. Dr. Khan Abul Kalam Azad, Professor of Medicine, Dhaka Medical College, Dhaka.

2. Dr. Md. Hafiz Sardar, Assistant Professor, Department of Medicine, Dhaka Medical College, Dhaka.

3. Dr. Susmita Hossain Natasha, Honorary Medical Officer, Department of Medicine, Dhaka Medical College Hospital, Dhaka.

4. Dr. Md. Uzzwal Mallik, Indoor Medical Officer, Department of Medicine, Dhaka Medical College Hospital, Dhaka.

5. Dr. Al Mahmood Appolo, Indoor Medical Officer, Department of Medicine, Dhaka Medical College Hospital, Dhaka.

6. Dr. Mohammad Shaiful Islam, Registrar, Department of Medicine, Dhaka Medical College Hospital, Dhaka.

Correspondence: Prof. Khan Abul Kalam Azad, Professor of Medicine, Dhaka Medical College, Dhaka.

Telephone: +880-2-8613252, Cell Phone: +8801711890508, Email: markazad24@yahoo.com 
while he was lying down. The seizure was generalized, affecting the whole body with jerky uncontrolled movement of all four limbs. It was associated with vomiting but not with tongue bite, or soiling of clothes. It persisted for 6-7 minutes and subsided spontaneously. There was no history of trauma, head injury, travelling to a malaria affected zone or contact with a TB patient. On further enquiry, attendants told that he was initially admitted to a local hospital and a CT Scan of Brain was done. The physicians told then that the patient has suffered from stroke and referred him to $\mathrm{DMCH}$. His bowel and bladder habit was normal. Though he could not speak but he could manage to convey his urges to through sign language. There was no subsequent seizure. He has no significant history of past illness. He was non smoker non alcoholic. He is the first issue of his parents. He has a healthy younger sister aged 9 years. He comes from a lower middle class family. He was allergic to aubergine. He was properly immunized as per EPI schedule. On examination the patient was found aphasic but he could understand what was being said to him. His blood pressure was $120 / 80 \mathrm{mmHg}$, pulse 84 beats/min, normal temperature, respiratory rate 22 breaths/ minute, Glasgow Coma Scoring was $\mathrm{E}_{4} \mathrm{~V}_{3} \mathrm{M}_{1}$. His pupils were bilaterally normal and reacting to light with normal fundoscopy. Examination of other cranial nerves revealed that he has UMNL in right facial nerve. Other cranial nerves were normal. His muscle power was found to be $1 / 5$ in right upper and lower limb. Reflexes were also diminished. Muscle tone was normal. Other systemic examination revealed no abnormality. His base line investigations consisting of a complete blood work, Peripheral Blood Film, ECG, Urine R/E, Serum Creatinine, Urea and Electrolytes, Random Blood Sugar all came back normal. His Bleeding time and clotting time were within normal range. But his Fasting Lipid Profile showed marked abnormality with a Serum Total Cholesterol being $196 \mathrm{mg} / \mathrm{dl}$, HDL Cholesterol 35 mg/dl, LDL cholesterol 105 mg/ $\mathrm{dl}$ and Serum triglyceride $277 \mathrm{mg} / \mathrm{dl}$. His Anti Nuclear Antibody, HBsAg, Anti HCV, Anti HIV all came back negative. A Trans esophageal
Echo revealed a small patent foramen ovale with left to right shunt. No vegetation or thrombus was noted. CT Scan of Brain (done before admission at $\mathrm{DMCH}$ ) revealed an acute, moderately large left fronto-temporal infarct and a chronic small left parietal infarct. Then a MRI of Brain was done and a zyriform intensity lesion was noted in left fronto-parietal region in $\mathrm{T}_{2}$ and FLAIR, which showed minimal enhancement after IV contrast. These features were suggestive of fibro lamelar cortical necrosis of left frontoparietal region and a differential diagnosis of Gliomatosis Cerebri was suggested. It may be mentioned here that Gliomatosis cerebri (infiltrative diffuse astrocytosis) is a rare primary brain tumor. It is commonly characterized by diffuse infiltration of the brain with neoplastic glial cells that affect various areas of the cerebral lobes. Gliomatosis cerebri behaves like a malignant tumor that is very similar to Glioblastoma. At this point a primary diagnosis of Intracranial Space Occupying lesion was made. Patient was empirically treated with steroid and anti-convulsant.

Two days after admission at $\mathrm{DMCH}$, patient was able to talk a little and within a week his speech returned with a residual slurring. He did not experience any further convulsions. His right upper and lower limbs also started gaining control and his muscle power started to return. As he could talk to us now, we took a detailed history from him. During this period he revealed that he had been having non-specific dull headaches for last three months and occasional visual difficulties that he could not elaborate properly. But this was his first episode of aphaisa, hemiplegia and convulsion. The aphasia and hemiplegia was sudden in onset, the convulsion followed afterwards. At this point a Digital Subtraction Angiogram was carried out to exclude vascular malformation. RICA angiogram reveals normal carotid bifurcation with small blushes around pericalossal artery. LICA Angiogram reveals discontinuation of left ICA at supraclenoid level. There were multiple Dural and Meningeal vessel supplying the left MCA and left ACA territory giving rise to the typical angiographic appearance of Moyamoya Disease. 
After the diagnosis the patient was treated conservatively with anti-convulsant and paracetamol. He followed up after a month for neurosurgical referral. During his stay at home he had another episode of seizure and right sided weakness that responded to conservative management. He still has some slurring of speech and residual disability in his right side of body. He has been also experiencing transient attacks of blindness and deafness that lasts up to 3 hours and then resolve spontaneously.

\section{Discussion:}

Moyamoya disease has a typical bimodal presentation with headache, seizure, ischaemic events and TIAs more common in young age and subarachnoid and intracerebral hemorrhages being more common after the fourth decade of life ${ }^{5}$.

A range of stimuli including brain radiation, injuries, or genetic defects, conditions such as sickle cell anemia, neurofibromatosis-1, Down's syndrome, congenital heart defects, antiphospholipid syndrome, renal artery stenosis, and thyroiditis have been found to be associated with Moyamoya disease in the literature ${ }^{6}$. Linkage was found with loci on chromosomes 3p24.2-26 and 17q25 and on chromosome 67 . The process of blockage, once it begins, tends to continue despite any known medical management (anti platelets have been used, anti coagulants have no role) unless treated with surgery ${ }^{8}$. MRI not only reveals areas of infarctions, but also allows direct visualization of these collateral vessels as multiple small flow voids at the base of brain and basal ganglia that is supplied by small perforating vessels from Circle of Willis. In Moyamoya disease, these perforating arteries enlarge due to the demand of the brain. In MR images, the enlarged perforating arteries are visualized as flow void (signal void) in the basal ganglia. Angiography is used to confirm the diagnosis and to see the anatomy of the vessels involved. It typically reveals the narrowing and occlusion of proximal cerebral vessels and extensive collateral flow through the perforating vessels demonstrating the classic puff of smoke appearance ${ }^{9}$.
Acute management is mainly symptomatic and directed towards reducing elevated intracranial pressure, improving cerebral blood flow, and controlling seizures. Revascularization procedures are currently performed to increase the perfusion to the hypoxic brain tissue. Studies support these procedures, and longterm favourable outcome has been reported in terms of improvement in symptoms and positive angiographic follow ups in all age groups ${ }^{10}$. Prognosis of patients with Moyamoya disease is found to be related to age and the type of presentation. Hemodynamic improvement after surgical procedures occurs irrelative of age. Patients presenting with TIAs or irritative brain lesions causing epileptiform clinical pictures have a better long-term outcome that patients with infarct ${ }^{11}$.

\section{Management:}

There are many operations that have been developed for the condition, but currently the most favored are the in-direct procedures EDAS, EMS, and multiple burr holes and the direct procedure STA-MCA. Direct superficial temporal artery (STA) to middle cerebral artery (MCA) bypass is considered the treatment of choice, although its efficacy, particularly for hemorrhagic disease, remains uncertain. Multiple burr holes have been used in frontal and parietal lobes with good neovascularisation achieved.

The EDAS (encephaloduroarteriosynangiosis) procedure is a synangiosis procedure that requires dissection of a scalp artery over a course of several inches and then making a small temporary opening in the skull directly beneath the artery. The artery is then sutured to the surface of the brain and the bone replaced.

In the EMS (encephalomyosynangiosis) procedure, the temporalis muscle, which is in the temple region of the forehead, is dissected and through an opening in the skull placed onto the surface of the brain.

In the multiple burr holes procedure, multiple small holes (burr holes) are placed in the skull to allow for growth of new vessels into the brain from the scalp. 
In the STA-MCA procedure, the scalp artery (superficial temporal artery or STA) is directly sutured to an artery on the surface of the brain (middle cerebral artery or MCA). This procedure is also commonly referred to as an EC-IC (External Carotid-Internal Carotid) bypass.

The perception behind all these procedure is quite simple. What these procedures do is to introduce a vasculature to oxygen 'starved' brain tissue and thus accelerates the physiological process of angiogenesis bypassing the areas of blockage. Stenosis or occlusion does not always mean cerebral ischemia. Thus, cerebral blood flow measurement is required to assess the real blood flow supplying the brain. In cerebral ischemia, small arteries in the brain may enlarge reactively to the demand. The degree of this reactive vasodilation is assessed by Acetazolamide loading which causes pharmacological vasodilation. SPECT (single photon emission computed tomography) and PET (positron emission tomography) are used for measurement of the cerebral blood flow. Preand post-operative $\mathrm{CBF}$ measurements are usually performed in the management of Moyamoya disease. The modified direct anastomosis and encephalo-myo-arteriosynagiosis play a role in this improvement by increasing cerebral blood flow (CBF) after the operation. A significant correlation is found between the postoperative effect and the stages of preoperative angiograms. It is crucial for surgery that the anesthesiologist have experience in managing children being treated for Moyamoya as the type of anesthesia they require is very different from the standard anesthetic children get for almost any other type of neurosurgical procedure.

\section{Conclusion:}

Moyamoya disease has poor prognosis without revascularization. The chances of recurrent stroke are obvious and eventually this leads to untimely death of the patient. But with proper revascularization the 5 year survival rate may be as high as $96 \%$ if the critical period of thirty days after surgery is passed uneventfully. In a country like Bangladesh where resources are limited, diagnosis of rarer diseases like Moyamoya disease may pose a challenge unto itself. The best way to approach such a case is with caution and open mind, excluding each possibility step by step. A battery of test may prove unfruitful if the thinking is not organized.

\section{References:}

1. Takeuchi K, Shimizu K. Hypogenesis of bilateral internal carotid arteries. No Ta Shinkai 1957; 9: 37-43.

2. Suzuki J, Takaku A. Cerebrovascular "moyamoya" disease: disease showing abnormal net-like vessels in the base of brain. Arch Neurol 1969; 20: 288-99.

3. Yamashita M, Oka K, Tanaka K. Histopathology of the brain vacular network in Moyamoya Disease. Stroke 1983; 14: 50-8.

4. Wetjen NM, Garell PC, Stence NV, Lopftus CM. Moyamoya disease in the midwestern United States. Neurosurg Focus 1998; 5(5): e1.

5. Llu XF,Jung DK. Moyamoya disease and migrainelike headaches. Schweiz Arch Neurol Psychiatr 1999; 150: $272-4$.

6. Lutterman J, Scott M, Nass R, Geva T. Moyamoya syndrome associated with congenital heart disease. Pediatrics 198; 101(1): 57-60.

7. Yamauchi T, Tada M, Houkin $\mathrm{K}$, et al. Linkage of familial moyamoya disease (spontaneous occlusion of the circle of Willis) to chromosome 17q25. Stroke 2000; 31: 930-5

8. Smith ER, Scott RM. Moyamoya syndrome associated with congenital heart disease. Skull Base 2005; 15(1): 15-26.

9. Atlas SW. MR angiography: techniques and clinical applications MRI of the brain and spine. $3^{\text {rd }}$ ed. Philadelphia: Lippincott Williams \& Wilkins; 2002.

10. Han DH, Kwon OK, Bark BJ, et al. A co-operative study: clinical characteristics of 334 Korean patients with moyamoya disease treated at neurosurgical institutes (1976-1994). Acta Neurochir 2000; 142(11):1263-74.

11. Inoue TK, Ikezaki K, Sasazuki T, Matsushima T, Fukui M. Linkage analysis of moyamoya disease on chromosome 6. J Child Neurol 2000; 15(3): 179-82. 